\title{
ASSISTÊNCIA TÉCNICA PARA CONSULTA AO CÓDIGO DE URBANISMO COM SUPORTE BIM/GIS
}

VISBQP

UBERLÂNDIA 2019

\author{
FARIAS JÚNIOR, Antonio Goncalves de \\ IFPB Cajazeiras, e-mail: antonio.farias@ifpb.edu.br \\ JERONYMO, Caroline Muñoz Cevada \\ IFPB Cajazeiras, e-mail: caroline.jeronymo@ifpb.edu.br \\ JERONYMO, Emanuel Lima Oliveira \\ IFPB Cajazeiras, e-mail: emanuel.jeronymo@academico.ifpb.edu.br
}

\begin{abstract}
RESUMO
A grande maioria das cidades promovem o acesso da população a legislação urbana através de uma linguagem textual jurídica o que pode dificultar o cidadão na compreensão sobre os índices urbanísticos (recuos, taxas de ocupação, alturas máximas) que incidem sobre seu terreno. Com suporte nas tecnologias Building Information Modeling - BIM e Geographic Information System - GIS abre-se um caminho para facilitar processos internos e burocráticos e a entrega dessas informações popularizando o acesso do cidadão. A partir de dissertação que desenvolveu um protótipo para João Pessoa, foi proposta a experiência da assistência técnica para consulta automática ao código de urbanismo com suporte nessas tecnologias para outra cidade: Cajazeiras/PB, a partir de edital de extensão aplicado por equipe de docentes e discentes do IFPB/Cajazeiras. Esse artigo tem como objetivo apresentar resultados desse trabalho na gestão do projeto através de uma equipe multidisciplinar que realizou metodologicamente atividades de consulta e análise a mapas, plantas cadastrais (impressas e digitais), tendo como resultado a produção de mapas temáticos, tabulação de dados numéricos descritos na legislação e estruturação de dados em modelos paramétricos para consultas automáticas, além da discussão sobre as dificuldades e barreiras para a implementação desses sistemas.
\end{abstract}

Palavras-chave: BIM, GIS, Legislação Urbana, Gestão de Projetos.

\begin{abstract}
The great majority of cities promote the access of the population to urban legislation through a juridical textual language which can make it difficult for the citizen to understand the urban indices (setbacks, occupancy rates, maximum heights) that affect his land. With support in Building Information Model (BIM) technologies and Geographic Information System (GIS), a way is opened to facilitate internal and bureaucratic processes and the delivery of this information, popularizing citizen access. From a dissertation that developed a prototype for João Pessoa, the experience of the technical assistance for automatic consultation to the code of urbanism with support in these technologies for another city was proposed: Cajazeiras / PB, from an extension notice applied by a team of teachers and students of IFPB Cajazeiras. This article aims to present results of this work in the project management through a multidisciplinary team that carried out methodologically activities of consultation and analysis to maps, cadastral plans (printed and digital), resulting in the production of thematic maps, tabulation of numerical data described in the legislation and data structure in parametric models for automatic queries, besides the discussion about the difficulties and barriers to the implementation of these systems.
\end{abstract}

Keywords: BIM, GIS, Urban Legislation, Project Management.

FARIAS JÚNIOR, A. G.; JERONYMO, C. M. C.; JERONYMO, E. L. O. Assistência técnica para consulta ao código de urbanismo com suporte BIM/GIS. In: SIMPÓSIO BRASILEIRO DE QUALIDADE DO PROJETO NO AMBIENTE CONSTRUÍDO, 6., 2019, Uberlândia. Anais... Uberlândia: PPGAU/FAUED/UFU, 2019. p 612-621. DOI https://doi.org/10.14393/sbqp19057. 


\section{INTRODUÇÃO}

O ano 2018 se tornou um marco para construção civil brasileira através do Decreto $n^{\circ}$ 9.377, que instituiu a estratégia nacional de disseminação do BIM com objetivo de promover um ambiente adequado ao investimento nessa tecnologia e sua difusão nacional (BRASIL, 2018). Compreendendo BIM como um conjunto de tecnologias e processos integrados que permite a criação e gerenciamento de modelos digitais durante o ciclo de vida da construção, estados e municípios deverão incluir ações que viabilizem a estruturação de processos de implantação dessa tecnologia a fim de alinhar a esse planejamento. Denominada Estratégia BIM-BR, essa ação do governo está dividida em alguns objetivos específicos como a estimulação ao desenvolvimento e aplicação de novas tecnologias relacionadas ao BIM. Já o A tecnologia GIS, já consolidada a mais tempo, é um sistema de hardware, software, de informação espacial, procedimentos computacionais e recursos humanos que permite e facilita a análise, gestão ou representação do espaço e dos fenômenos que nele ocorrem. Um fator que contribuiu para a disseminação de dados geográficos pela internet foi o baixo custo empregado fazendo com que hoje, o software GIS já possua alternativas de acesso de dados via web (DAVIS JÚNIOR, SOUZA; BORGES, 2005). Há inclusive software livre como o Qgis, na terceira versão e já traduzido para o português na maioria dos comandos.

Considerando as duas tecnologias, uma dissertação em desenvolvimento no Programa de Pós-Graduação de Arquitetura e Urbanismo da Universidade Federal da Paraíba (PPGAU-UFPB) buscou aliar em um protótipo, a experiência de consulta a índices e dados de legislação urbanística da cidade de João Pessoa-PB, a fim de facilitar o acesso a dados pela população. No protótipo foi desenvolvido um sistema que permite consulta automática ao Código de Urbanismo da capital paraibana, sendo este passível de aplicação a cidades que possuam banco de dados cartográficos e valores numéricos para os índices urbanísticos.

Experimentou-se aplicar o protótipo na cidade de Cajazeiras-PB durante projeto de extensão na modalidade "assistência técnica" viabilizado através de uma cooperação entre três entidades: 1. o Laboratório de Modelagem e Prototipagem (LM+P-UFPB), que cedeu e apresentou as funcionalidades e aplicações do protótipo do mestrado acadêmico (FARIAS JÚNIOR, 2017); 2. a equipe técnica heterogênea montada pelo IFPB Cajazeiras, com outros docentes e discentes; e 3. a participação da Prefeitura Municipal de Cajazeiras, atuando como beneficiária. Relatar o trabalho de assistência técnica para implantação dessas tecnologias no processo de consulta do código urbano com estudo de caso em Cajazeiras-PB é o objetivo deste artigo.

Vale destacar que essa experiência corresponde à implantação de um sistema que busca diversificar o acesso a legislação urbana a população sendo capaz de entregar informações de um modo que visa ampliar a compreensão do cidadão sobre os índices urbanísticos como por exemplo recuos, taxas de ocupação, alturas máximas, que incidem sobre seu terreno. Além de facilitar processos internos e burocráticos, com o avanço deste projeto poderemos popularizar informações, tornando-as mais simples a sua compreensão e acessíveis ao cidadão comum de Cajazeiras, que nem sempre tem o domínio da linguagem técnica das legislações. 
Até o momento, O BIM "continua sendo uma ferramenta relativamente subutilizada na construção horizontal", apesar do seu potencial de "aumentar significativamente a eficiência, minimizar $o$ desperdício e aumentar a sustentabilidade" em projetos de infraestrutura ao longo de seus ciclos de vida (FANNING et al., 2014, p. 4). Fanning et al. (2014, p. 8) também apresentam descobertas adicionais que sugerem que o uso do BIM pode facilitar projetos complexos de outra forma inviáveis e reduzir pedidos de informações e pedidos de mudança, além de ter o potencial de reduzir os custos durante os projetos subsequentes.

O BIM pode atuar com outras tecnologias em várias fases de um projeto, permitindo a atuação de diferentes atores envolvidos. Duas das barreiras recorrentes apresentadas no processo de implantação da tecnologia BIM são a falta de cultura BIM entre os envolvidos e os desafios de integração e interoperabilidade com outras tecnologias, a exemplo do GIS.

Esse relato é pertinente inclusive, por tratar também da experiência de integração entre estes sistemas aplicado a um trabalho com equipe multidisciplinar apresentando sua primeira experiência prática na organização e aplicação de método BIM-GIS operando na legislação urbana, sob a hipótese de que o protótipo desenvolvido poderia ser replicado e, que a assistência técnica seria eficaz na facilitação e acesso da comunidade local aos dados e índices urbanísticos.

\section{FUNDAMENTAÇÃO}

A implementação do BIM já nas primeiras etapas de desenvolvimento do projeto pode representar avanços para a tomada de decisões mais consistentes e em menor tempo e custo de produção (EASTMAN et al., 2014, p. 99). Segundo Brasil (2010), em cartilha desenvolvida para o Ministério das cidades, um fator que contribuiu para o aumento da demanda de serviço das prefeituras nos últimos anos foi a criação do programa Minha Casa Minha Vida, que só no triênio de 2009/2011 tinha como meta incentivar a produção e compra de um milhão de moradias. Criar mecanismos que otimizem a etapa de consulta ao banco de dados da prefeitura permitirá uma economia de recursos público uma vez que o processo de automação proporciona redução de tempo investido nas atividades permitindo ganho de produtividade. No contexto da produção do projeto arquitetônico, Eastman et al. (2009) dizem que a longo prazo serão requeridos métodos de validação de regras codificadas e a validade e consistência da modelagem de informações do edifício. Solihin e Eastman (2015) definem que cada sistema já poderia ser aplicado imediatamente numa ampla base na indústria da construção civil.

Um exemplo de consulta automática a mapas cartográficos que já vem sendo aplicado é o E-SIG - Sistemas de Informações Geográfica mantido pela prefeitura da cidade do Recife/PE. De livre acesso ao público, o sistema foi criado para disseminar informações georreferenciadas do município, permitindo download de dados para serem usados em ferramentas SIG. Mas também é destacada suas vantagens por permitir uma alimentação de dados que podem ser coletados juntos a outros órgãos da administração pública permitindo combinações de diversas informações em estruturas tabulares. 
Outro conceito a ser fundamentado é o de modelo paramétrico que de acordo Eastman et al, estaria diretamente relacionado a programação do comportamento de objetos:

\begin{abstract}
Conceitualmente, ferramentas de Modelagem da Informação da Construção (BIM) são modelos paramétricos baseados em objetos com um conjunto predefinido de famílias de objetos; cada uma tem comportamentos programados dentro deles [...]Uma configuração de modelo de construção é definida pelo usuário como uma estrutura paramétrica dimensionalmente controlada [...] existe um amplo conjunto de práticas padrão e códigos que podem ser prontamente adaptados e embutidos para definir os comportamentos dos objetos (EASTMAN et al., 2014, p. 33).
\end{abstract}

Dessa forma, estando aparelhado das regras que definiriam o comportamento de um objeto para um determinado fim, seria possível produzir um modelo BIM capaz de reproduzir esse comportamento, tendo como característica comunicar o resultado através da modelagem 3D com recurso de visualização. Através de Hudson, observamos o modelo paramétrico definido como um fenômeno do conhecimento sobre o campo heurístico:

[...] qualquer tipo de procedimento principal ou de outros dispositivos que possa reduzir a busca para uma solução satisfatória [...]. A identificação dos parâmetros-chave (aqueles que apresentam o melhor efeito no desenho) dentre vários parâmetros reduz o volume de pesquisa [...]. Aqui se propõe que as ferramentas paramétricas podem fornecer a representação para capturar o conhecimento existente e adquirir novos. O processo de aquisição de conhecimento é observado como equivalente a exploração. [...] com o sentido de descobrir novas funcionalidades, restrições e parâmetros e sugerindo como a descrição de um problema existente pode ser ajustado ou descartado (HUDSON, 2009 apud NEWELL; SHAW; SIMON, 1957, tradução nossa).

Destarte, o desenvolvimento do protótipo buscou explorar a potencialidade da tecnologia BIM, utilizando documentos e produtos da legislação urbana, que, rigorosamente, deveria ser de abrangência e usabilidade pública e coletiva. O processo de implementação da ferramenta em qualquer município, no entanto, depende da cooperação dos técnicos da prefeitura lotados nos setores Planejamento e infraestrutura em geral. Apesar da Lei 12.527/201 1 (Lei Acesso à Informação) e da Lei 131/2009 (Lei da Transparência), permitir à equipe de implantação acesso a documentos de legislação urbana (Código de Obras, Plano Diretor, ou documentos equivalentes), às entrelinhas de alguns destes documentos, que por vezes desatualizados, ou então, com uma frequente rotina de revisões, por vezes com linguagem pouco acessível; a relação direta e formal com agentes da prefeitura é imprescindível. Quando disponibilizado por órgão municipal competente, o mapa do loteamento da região de interesse, através de imagem de satélite e/ou foto da área é utilizada para ajustes.

O método de consulta paramétrica do sistema proposto pelo protótipo que será usado como base para esse trabalho se baseia na automação e 2 tarefas que acontecem durante a consulta os índices urbanísticos: A localização da zona urbana e o cálculo de áreas do potencial construtivo. Para cada lote é criado uma shapefile correspondente carregados com atributos relacionados 
ao zoneamento. Quando o Usuário na plataforma webGIS localiza o seu terreno, as coordenadas geográficas são enviadas para o sistema do protótipo, que carrega os atributos, e partir do desenho do usuário e informações da finalidade construtiva gera no output relatório e arquivos editáveis (Figura 1).

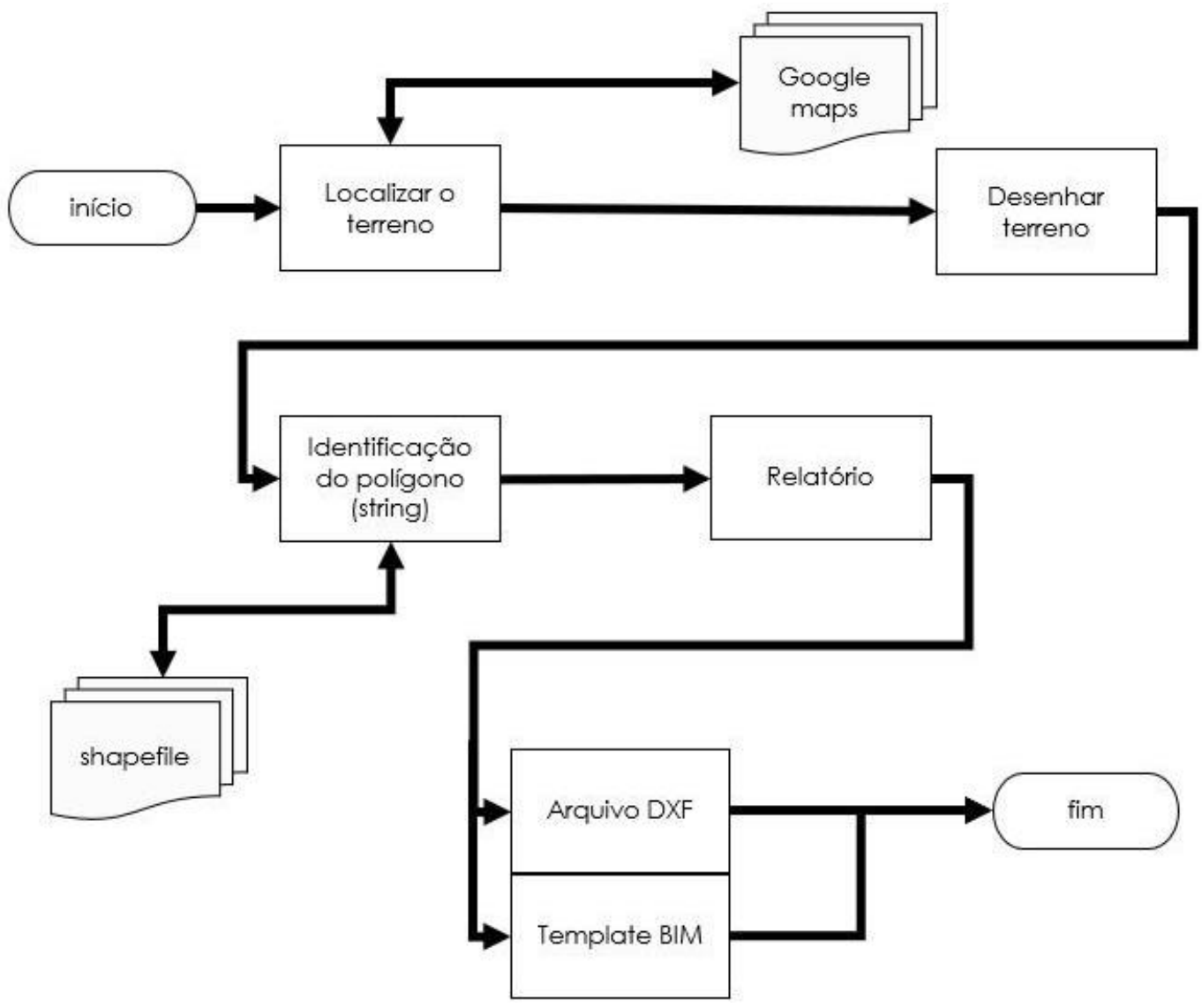

Figura 1 - Fluxograma esquemático do Protótipo WEB-GIS-BIM Fonte: FARIAS JÚNIOR (2017)

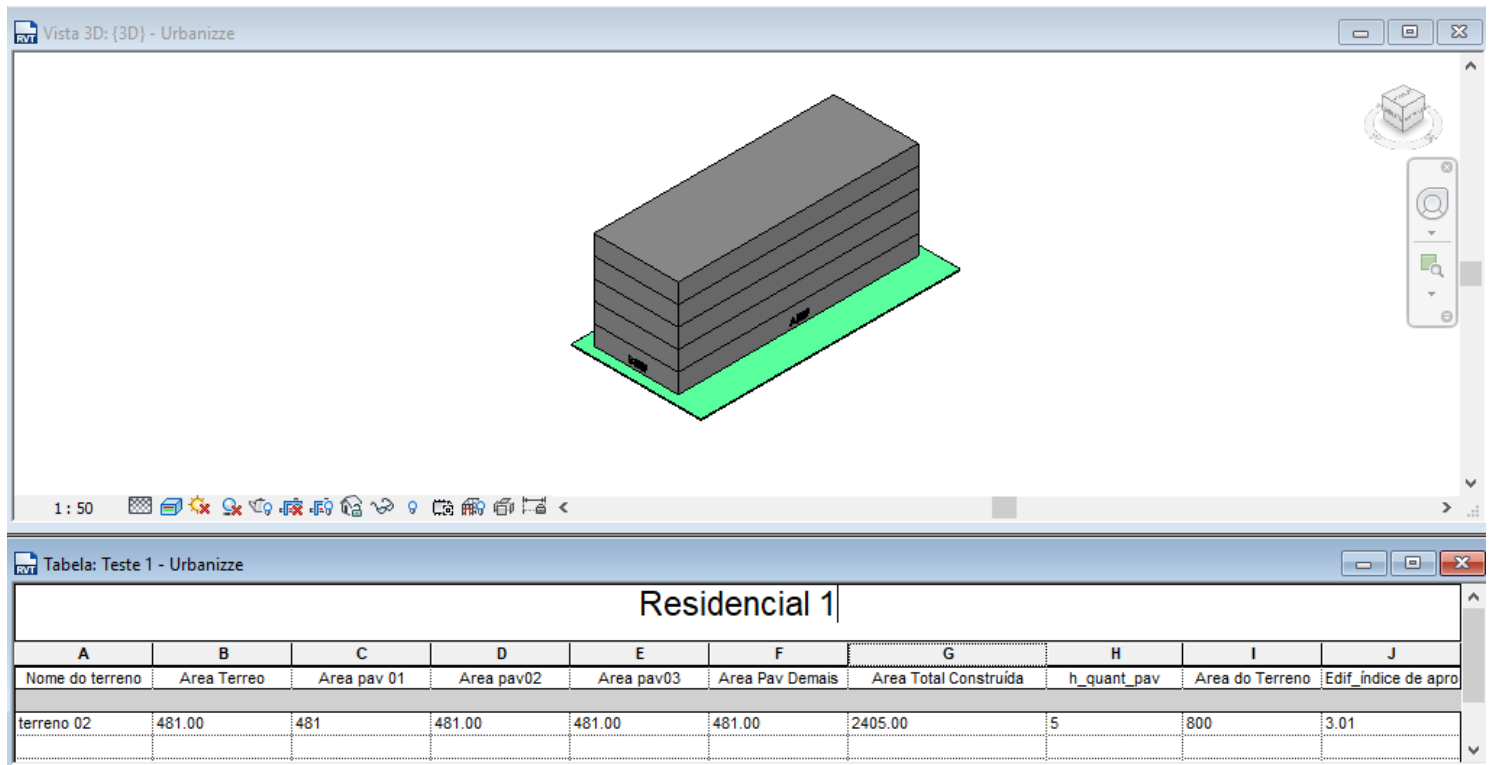

Figura 2 - Exemplo de resultado gerado no Template BIM, a partir de consulta de terreno específico dentro do processo do protótipo -

Fonte: FARIAS JÚNIOR (2017) 
Um template BIM é o produto tridimensional, com as medidas equivalentes às informações da zona urbana localizada. A partir da topografia planimétrica do terreno urbano, a família apresenta os dados coletados na legislação urbana municipal e materializa visualmente índices como recuos aplicados ao uso do solo definido. A Figura 2 mostra a interface do Revit onde é onde o modelo é manipulado e a tabela de dados que é gerado como relatório final.

No entanto, é válido salientar que o protótipo não pretende substituir a atuação de profissionais da construção civil no seu processo criativo de concepção volumétrica. O protótipo tem caráter consultivo e exerce a função de suporte a estudos de viabilidade construtiva.

\section{METODOLOGIA}

A pesquisa é de natureza exploratória pois investiga, em um estudo de caso único, as possibilidades e experiências da aplicação do protótipo na assistência técnica da implantação da tecnologia BIM e GIS em cidade do interior paraibano.

Para a implantação da assistência técnica foi constituída equipe que integrasse o conhecimento de diversas áreas: Administração, Engenharia Civil, Urbanismo, técnico em Edificações, técnico em Informática e Gestão Ambiental; característica necessária para a construção da pesquisa, já que estes sistemas de informação complexos transitam por vários saberes e aplicações.

Considerando a experiência de prática inovadora para os envolvidos e o curto prazo de aplicação e desenvolvimento (de outubro a dezembro de 2018), foram escolhidos métodos ágeis de aplicação, por serem "indicados para cenários em que existe constante mudança de requisitos e os resultados devem ser entregues em pequenos espaços de tempo" (CARVALHO; MELLO, 2012). Optou-se pelo método SCRUM de gerenciamento de projetos, com uso da ferramenta Trello, que se baseia no método Kanban (JHONSON, 2017). O Trello permitiu a interação de todos os agentes integrantes da execução, facilitando o contato, organizando e acompanhando a dinâmica das atividades, podendo, durante os ciclos de prazo previstos (sprints), sugerir, redirecionar, corrigir e isolar os impedimentos.

As etapas metodológicas foram caracterizadas por:

- Capacitação dos envolvidos para atuação BIM/GIS;

- Análise documental - reunindo mapas mais atualizados e disponíveis para utilizar como base para a atualização de mapa georreferenciado;

- Contratação de hospedagem e serviço de base para site, para lotar o protótipo online;

- Consulta a mapas e plantas cadastrais (impressas, digitais - GIS);

- Análise e "tradução" dos dados encontrados no Código de Obras da cidade em estudo por meio de tabulação das variáveis do código a serem automatizadas;

- Produção dos shapefile das zonas urbanas, a partir dos mapas encontrados;

- Extração das variáveis mediante análise dos documentos de legislação urbana municipal; 
- Implementação dos dados na plataforma WEB.

Sinteticamente, é possível compreender estas etapas em 04 Fases: 1. Início; 2. Coleta de dado; 3. Produção; e 4. Implantação (Figura 3).
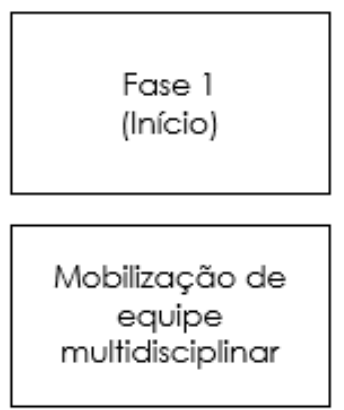

\begin{tabular}{|c|}
\hline Comunicação \\
com equipe da \\
Prefeitura \\
Municipal \\
\hline
\end{tabular}
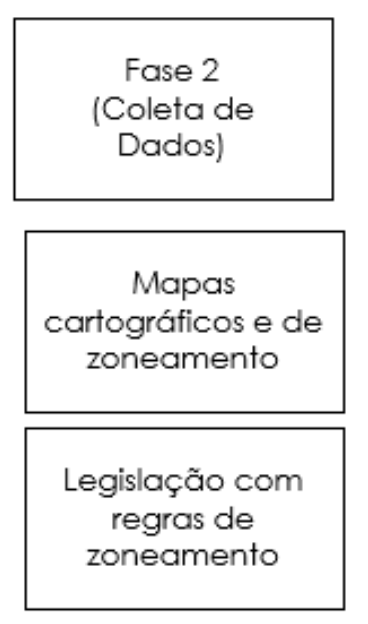
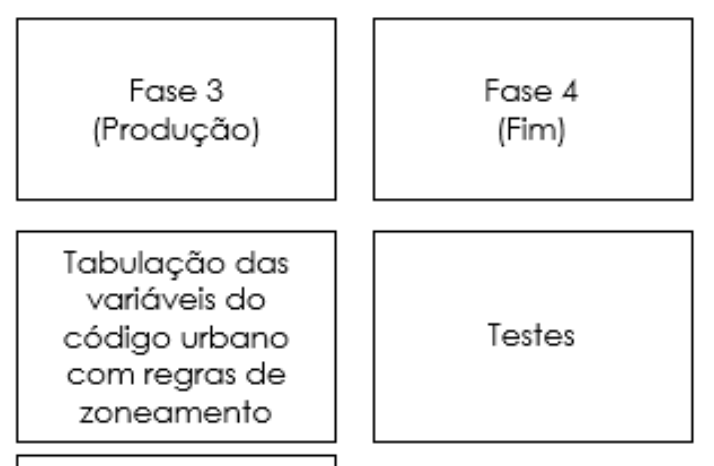

Produção dos shapefiles das zonas urbanas

Integração ao ambiente web

\section{Figura 3 - Síntese dos procedimentos metodológicos previstos para a execução da assistência técnica proposta - Fonte: Autores (2019)}

\section{RESULTADOS E DISCUSSÃO}

Dentro do prazo estabelecido para a execução do edital de extensão e da implementação do protótipo na cidade objeto de estudo houveram algumas limitações e percalços, como desenvolver nos alunos a capacidade de gestão da informação a fim de dar retorno a produção realizada um determinado período do serviço, cumprir o cronograma das atividades em um curto período de tempo e o trabalho com a base de dados (documentos da legislação urbana de responsabilidade externa) de difícil compreensão.

No entanto, a implantação do protótipo permitiu a troca de experiências e conhecimento acadêmico tecnológico com a equipe do Laboratório LM+PUFPB e equipe IFPB-Cajazeiras, possibilitou $O$ aprofundamento do conhecimento adquirido pelos alunos sobre as tecnologias BIM e GIS, aproximou e facilitou o contato com da equipe acadêmica com setores da Prefeitura Municipal de Cajazeiras-PB que prestam serviços de aprovação de projetos de arquitetura sob o olhar da legislação urbana.

Os sprints previstos materializaram alguns produtos: como o desenho do fluxograma operacional, construção do backend do ambiente web, atualização do mapa de Cajazeiras (shapefile) (Figura 4).

Durante a consulta a mapas e plantas cadastrais (impressas e digitais), foi percebido que o traçado apresentado estava desatualizado, e foi ajustado no software GIS disponível a partir de imagens de satélite georeferenciadas. A 
atualização produziu arquivos no formato shapefile das faces e logradouros e limite municipal.

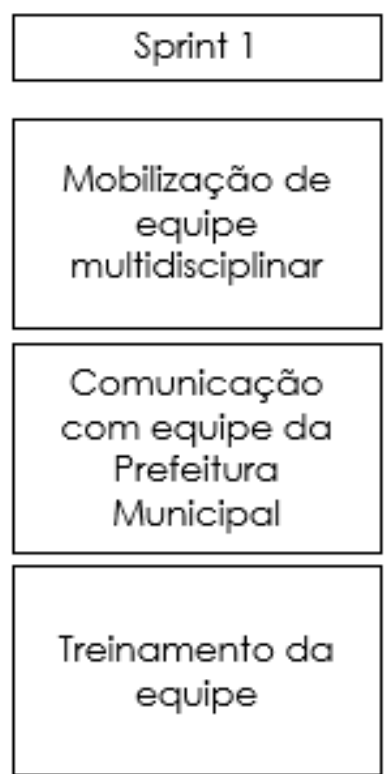

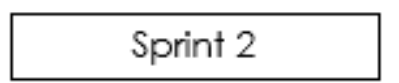

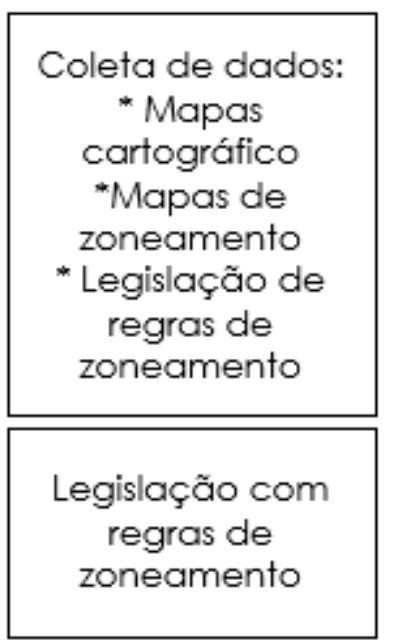

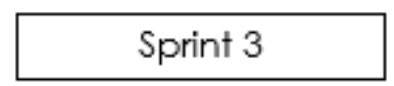

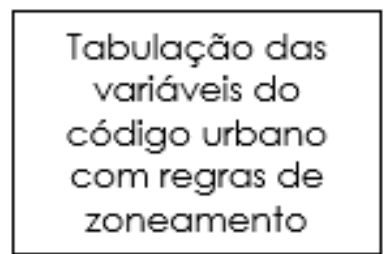

Produção dos shapefiles das zonas urbanas

Integração ao ambiente web

Figura 4 - Síntese dos sprints realizados na assistência técnica -

Fonte: Autores (2019)

$\mathrm{Na}$ etapa seguinte foram verificados os documentos de legislação urbana municipal, para a produção da tabulação de dados numéricos dos índices urbanísticos descritos ao longo dos documentos normativos, e estruturação de dados para emissão dos relatórios e das consultas automáticas. A organização e tabulação de índices urbanísticos do Código de Urbanismo e Obras da cidade representou para os profissionais do setor de provação da Prefeitura Municipal já um avanço para a compreensão do código uma vez que o mesmo não possuía tabelas de zoneamento a exemplo de outras cidades.

Com os dados tabulados, foi dado início a parametrização das variáveis no ambiente BIM. Essa atividade foi parcialmente concluída devido a limitações técnicas que surgiram na equipe durante o seu desenvolvimento.

Vale ressaltar que o ambiente BIM objetiva atender inicialmente apenas profissionais da construção civil que utilizem o Revit, pois foi o software escolhido para o desenvolvimento. Já a parte do sistema ancorado no ambiente webGIS tem acesso universal e onde os relatórios gerados, permitem a qualquer parte interessada fazer seu uso.

\section{CONSIDERAÇÕES FINAIS}

Com o desenvolvimento deste projeto a partir de serviço prestado, foi possível perceber que foi desenvolvida a autonomia dos discentes através da resolução de problemas reais em ambiente multidisciplinar. Esse processo foi expresso pela dinâmica entre pares e entre o grupo de forma heterogênea, geridos pela interação da ferramenta de gestão de projeto.

Além do aspecto positivo da formação continuada, a interação com o beneficiário do projeto - o arquiteto da Prefeitura Municipal, somou 
contribuições acerca do projeto e das limitações locais, direcionando um desenvolvimento aprofundado no uso das tecnologias.

O serviço prestado foi parcialmente concluído, e o desenvolvimento de suas atividades teve seu prazo dilatado, deixando o uso do protótipo para próximos editais. A hipótese sobre as dificuldades encontradas para conclusão pode estar relacionados ao tempo de execução que não permitiu a maturação da equipe técnica no uso das tecnologias BIM e webGIS, a heterogeneidade da equipe pode ter sido outro fator que colaborou para as dificuldades de coordenação. Contudo, esta experiência libera potencial para estudos focados na área de integração entre webGIS/BIM, e na aplicação da tecnologia desenvolvida pela academia para resolução de problemas recorrentes e reais.

\section{AGRADECIMENTOS}

Agradecemos ao fomento do edital $N^{\circ} 011 / 2018$ do Programa de Apoio Institucional à Prestação de Serviço do IFPB Campus Cajazeiras; à contribuição dos discentes/docentes participantes do IFPB: Higor Damaceno, Maria Vitoria Batista, Abraão Lira, Antônio Medeiros, Bruno Ramalho e profs. Marcello Benigno e Wilma Pinheiro; ao laboratório de Modelagem e Prototipagem LM+P/PPGAU/UFPB; também aos parceiros sociais que possibilitaram a heterogeneidade do grupo em uma rede cooperativa ministrando minicursos de capacitação: profa. Alexsandra Rocha, do Laboratório Cartografia e Geoprocessamento - LACAGEO/UFCG e Francisco Thiago Cavalcanti, da Faculdade Santa Maria.

\section{REFERÊNCIAS}

BRASIL. Lei Complementar no 131, de 27 de maio de 2009. Acrescenta dispositivos à Lei Complementar no 101, de 4 de maio de 2000, que estabelece normas de finanças públicas voltadas para a responsabilidade na gestão fiscal e dá outras providências, a fim de determinar a disponibilização, em tempo real, de informações pormenorizadas sobre a execução orçamentária e financeira da União, dos Estados, do Distrito Federal e dos Municípios. Lei da Transparência. 1. ed. Brasília, DF: Governo Federal, 27 maio 2009. Disponível em: <http://www.planalto.gov.br/ccivil_03/leis/lcp/lcp131.htm>. Acesso em: 27 mar. 2019.

Como produzir moradia bem localizada com recursos do programa minha casa minha vida?: Implementando os instrumentos do Estatuto da Cidade. Organização de Raquel Rolnik. Textos de Raphael Bischof e Joyce Reis. Brasília: Ministério das Cidades, 2010.

Regula o acesso a informações previsto no inciso XXXIII do art. $5^{\circ}$, no inciso II do $\S 3^{\circ}$ do art. 37 e no $\S 2^{\circ}$ do art. 216 da Constituição Federal; altera a Lei $n^{\circ} 8.112$, de 11 de dezembro de 1990; revoga a Lei $n^{\circ} 11.111$, de 5 de maio de 2005, e dispositivos da Lei no 8.159, de 8 de janeiro de 1991; e dá outras providências. Lei de Acesso à Informação. 1. ed. Brasília, DF: Governo Federal, 18 nov. 2019. Disponível em: <http://www.planalto.gov.br/ccivil_03/_ato201 1 2014/2011/lei/l12527.htm>. Acesso em: 27 mar. 2019.

DECRETO No 9.377. Institui a Estratégia Nacional de Disseminação do Building Information Modelling, Diário Oficial da União. Brasília. 2018. 
CARVALHO, B. V.; MELLO, C. H. P. Aplicação do método ágil scrum no desenvolvimento de produtos de software em uma pequena empresa de base tecnológica. Gestão \& Produção, [s.l.], v. 19, n. 3, p.557-573, 2012. FapUNIFESP (SCIELO).

DAVIS JÚNIOR, C. A.; SOUZA, L. A.; BORGES, K. A. V. Disseminação de dados geográficos na Internet. In: Banco de dados geográficos. Curitiba: MundoGeo, 2005.

EASTMAN, C. et al. Automatic rule-based checking of building designs.

Automation in Construction, v. 18, n. 8, p. 1011-1033, 2009.

Manual do BIM - Um guia de modelagem da informação da construção para arquitetos, engenheiros, gerentes, construtores e incorporadores. Porto Alegre: Bookman, 2014.

FANNING, B. et al. Implementing BIM on Infrastructure: Comparison of Two Bridge Construction Projects. Practice Periodical On Structural Design And Construction, [s.l.], v. 20, n. 4, p. 01-8, nov. 2014.

FARIAS JÚNIOR, A. G. O que é possível construir no seu terreno: Protótipo para verificação automática para ensaios de estudos de viabilidade arquitetônica em conformidade com parâmetros urbanos. In: BANCA DE QUALIFICAÇÃO, 20171., 2017, João Pessoa. Relatórios de Qualificação. UFPB: PPGAU, 2017. p. 1 70.

HUDSON, R. Parametric Development of Problem Descriptions. v. 07, n. 02, p. 199-216, [s.d.], 2009.

SOLIHIN, W.; EASTMAN, C. Classification of rules for automated BIM rule checking development. Automation in Construction, v. 53, p. 69-82, 2015. 